\title{
Performance Evaluation of a 3-Level ANPC Photovoltaic Grid-Connected Inverter with 650V SiC Devices and Optimized PWM
}

\author{
Davide Barater, Member, IEEE, Carlo Concari, Member, IEEE, Giampaolo Buticchi, Emre Gurpinar, Student \\ Member, IEEE, Dipankar De, Member, IEEE, and Alberto Castellazzi
}

\begin{abstract}
Photovoltaic (PV) energy conversion has been on the spotlight of scientific research on renewable energy for several years. In recent years the bulk of the research on PV has focused on transformerless grid-connected inverters, more efficient than traditional line transformer-based ones, but more critical from a power quality point of view, especially in terms of ground leakage current. Neutral point clamped (NPC) inverters have recently gained interest due to their intrinsically low ground leakage current and high efficiency, especially for MOSFET-based topologies. This paper presents an active NPC (ANPC) topology equipped with $650 \mathrm{~V}$ SiC MOSFETs, with a new modulation strategy that allows to reap the benefits of the wide-bandgap devices. An efficiency improvement is obtained due to the parallel operation of two devices during the freewheeling intervals. Simulations and experimental results confirm the effectiveness of the proposed converter.
\end{abstract}

\section{INTRODUCTION}

In recent years, an increment of production from renewable resources was registered in the energy mixes of several countries. Among renewables, the photovoltaic (PV) represents one of the most mature technologies. One of the major characteristics favoring PV over other renewable resources is the possibility to be employed in low power plants, easily integrated in the environment and directly connected to the low voltage distribution line. Many types of single-phase PV inverters were developed, both in industry and in academia [1]-[5]. Transformerless inverters are those that can achieve the highest efficiency levels, but their use in grid-connected systems is not straightforward.

In fact, due to the absence of galvanic isolation, undesired phenomena, such as ground leakage currents, can arise in

\footnotetext{
Manuscript Received Xxx yy, zzzz. Revised Xxxx yy, zzzz. Accepted for publication Xxxx y, zzzz. Copyright (c) 2015 IEEE. Personal use of this material is permitted. However, permission to use this material for any other purposes must be obtained from the IEEE by sending a request to pubspermissions@ieee.org.

D. Barater and C. Concari are with the Department of Information Engineering, University of Parma, Italy. e-mail: (davide.barater@nemo.unipr.it, carlo.concari@unipr.it).

G. Buticchi is with Chair of Power Electronics, Christian-Albrechts University of Kiel, 24143 Kiel, Germany. e-mail: (giampaolo.buticchi@unipr.it).

Emre Gurpinar and Alberto Castellazzi are with the PEMC Group at the University of Nottingham, UK. e-mail: (Emre.Gurpinar@nottingham.ac.uk, Alberto.Castellazzi@nottingham.ac.uk).

Dipankar De is with the Ryerson University, Toronto, ON, Canada. e-mail: (dde@ee.ryerson.ca).
}

transformerless plants [6]. Different architectures of inverters that address this issue were presented in literature; therefore transformerless inverters can be subdivided in two major families: full-bridge based and half-bridged based topologies [7]. The latter have the disadvantage of needing twice the value of the DC source voltage respect to the full-bridge based family, but, on the other hand, may achieve better performance in terms of ground leakage current rejection. The Neutral Point Clamped (NPC) converter architecture belongs to the family of half-bridge/based converters; it was first proposed in [8] for three-phase systems, but has been largely studied also for PV single-phase transformerless applications.

The structure of a single-phase NPC converter is shown in Fig. 1(a)). It relies on 4 switches and 2 diodes, synthesizing a three-level output voltage waveform. In [9] the diodes were replaced by power switches, realizing the so-called Active Neutral Point Clamped converter, ANPC (Fig. 1(b)). Threelevel ANPC claims better loss distribution and equal stress across the devices in comparison to conventional three-level NPC. Several PWM patterns and architectural variations were proposed for ANPC converters. In [10] an auxiliary circuit was added to the NPC structure to achieve soft switching commutation. A modulation technique was proposed in [11] for doubling the apparent switching frequency of the output voltage. A feedback controlled loss balancing system has been proposed for ANPC converters in [12]. In this case an online estimation of the devices' junction temperature was used to select the appropriate switching technique for reducing the switching stress of the hottest devices.

Moreover, in [13] the authors introduced the concept of the stacked neutral point clamped (SNPC) converter, in which an additional branch, constituted by two anti-series devices, is inserted between the converter output and the mid-point of the DC source (Fig. 2(a)). The concept was enhanced in [14], enabling a better loss distribution by substituting diodes with power switches (active SNPC, ASNPC) (Fig. 2(b)). In [15] a high-efficiency three-level SNPC architecture, using hybrid CoolMos and IGBT power modules, was proposed and applied to nonisolated grid-tied PV converters.

All the solutions above were limited to the use of silicon devices, and were optimized for IGBTs as well as for MOSFETs. In the latter case the modulations were optimized to avoid the conduction of the antiparallel MOSFET body diodes, therefore the active rectification capabilities of the MOSFETs was not yet exploited. 
This paper proposes an improved modulation to exploit the emerging silicon carbide devices. In order to take advantage of the low conduction losses and resistive on-state characteristic of the MOSFETs, the parallel conduction of the MOSFETs will be used during the freewheeling phases of the output current. This can be achieved employing silicon carbide ( $\mathrm{SiC}$ ) instead of silicon devices. SiC MOFETS have negligible antiparallel diode reverse recovery time, therefore the diodes can effectively carry the current during the dead time intervals.

A version of this paper was presented at the ECCE 2014 conference [16]. The paper has been extended and improved, and an extensive power loss analysis has been added.

\section{State of the ART AND Proposed High-EfFiciency PWM STRATEGY}

The original NPC converter structure is reported in Fig. 1(a). The DC link is composed of two series capacitors that share equal voltage, with $V_{D C}=V_{C 1}+V_{C 2}$. The neutral wire of the grid is connected to the mid-point of the DC voltage source, whereas the phase wire is connected to the filter inductor. It presents only three commutation states: P, 0 and $\mathrm{N}$ in Table I. Switches $S_{1}$ and $S_{2}$ commutate complementary to $S_{1 C}$ and $S_{2 C}$ respectively. During the positive half-wave of $v_{\text {grid }}, S_{2}$ is always ON whereas $S_{1}$ and $S_{1 C}$ commutate at high frequency (switching frequency $f_{s w}$ ). On the contrary, when $v_{\text {grid }}$ is negative, $S_{1 C}$ is $\mathrm{ON}$ and $S_{2}$ and $S_{2 C}$ commutate complementary at $f_{s w}$. Therefore, when $S_{1}, S_{2}$ are ON the load is directly connected to the DC source and $v_{\text {out }}$ is equal to $V_{D C} / 2$ (state P). In the same way, during state $\mathrm{N}, S_{1}, S_{2}$ are turned $\mathrm{OFF}$ and $v_{\text {out }}=-V_{D C} / 2$. In 0 state both the inner switches, $S_{2}$ and $S_{1 C}$, are $\mathrm{ON}$ and the load current (grid current) can pass through two different paths depending on its sign: positive grid current passes through $D_{1}$ and $S_{2}$, while the negative grid current passes through $D_{2}$ and $S_{1 C}$.

Using this modulation technique, for each device the average switching frequency over a $v_{\text {out }}$ period $\left(f_{a v}\right)$ is equal to $f_{s w} / 2$, while the apparent switching frequency of the output voltage is $f_{a p}=f_{s w}$.

The power loss distribution among the devices is highly unbalanced and strongly dependent on the operating point and on the PWM strategy adopted [11]. For low values of the PWM duty cycle the most stressed devices are the inner switches and diodes, while for high value of the duty cycle the outer switches $\left(S_{1}, S_{2 C}\right)$ present the highest conduction losses.

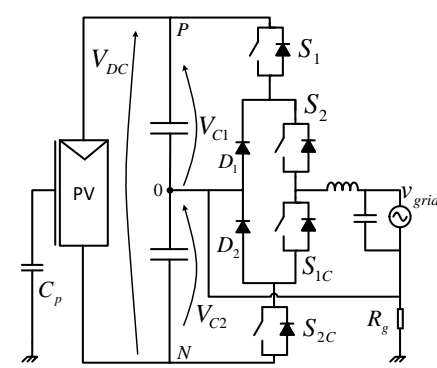

(a)

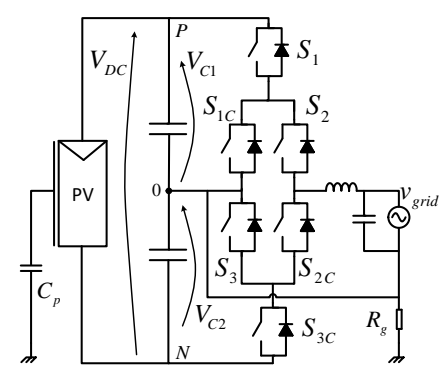

(b)
Fig. 1. NPC (a) and ANPC (b) in PV grid-tied transformerless applications.

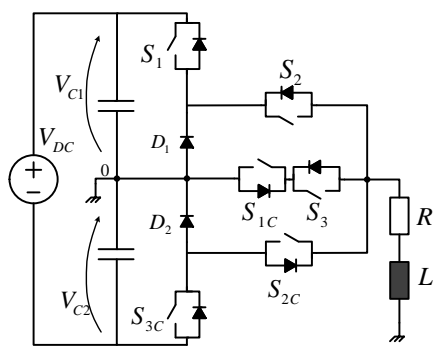

(a)

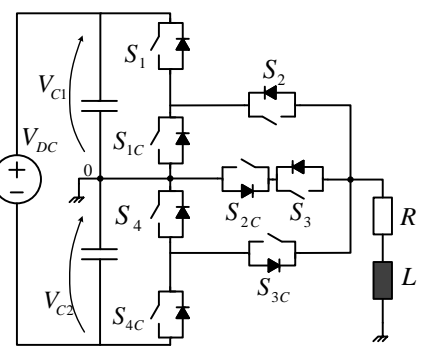

(b)
Fig. 2. SNPC (a) and ASNPC (b) architectures.

TABLE I. SWITCHING SEquences of A NPC CONVERTER

\begin{tabular}{|c|c|c|c|c|c|}
\hline Output & Switching & \multicolumn{4}{|c|}{ Switching Sequence } \\
\cline { 3 - 6 } Voltage & State & $S_{1}$ & $S_{1 C}$ & $S_{2}$ & $S_{2 C}$ \\
\hline$V_{D C} / 2$ & $\mathrm{P}$ & 1 & 0 & 1 & 0 \\
\hline 0 & 0 & 0 & 1 & 1 & 0 \\
\hline$-V_{D C} / 2$ & $\mathrm{~N}$ & 0 & 1 & 0 & 1 \\
\hline
\end{tabular}

The unequal distribution of the power losses among the devices is the main drawback of the NPC topology, and its effects are particularly severe in case of medium and high power applications [17]. The ANPC converter was proposed to overcome this issue (Fig. 1(b)). Using power switches instead of diodes increases the number of degrees of freedom for the control, since it is possible to obtain more than one zero state. This redundancy can be exploited to distribute losses more evenly among the devices, as well as to improve the efficiency of the converter. It has to be noted that equalizing the loss distribution among the power devices is not the main focus of this paper. Although a better loss distribution was obtained relative to NPC, the modulation pattern proposed in this paper is mainly focused on loss reduction, as will be clear in the following.

Several PWM techniques were proposed for ANPC converters in [11]. Among them, an interesting solution doubles the apparent switching frequency of the converter output voltage $\left(f_{a p}=2 f_{s w}\right)$. This modulation is taken in this paper as a benchmark to evaluate the performance of the proposed improved PWM pattern against. Employing the technique in [11] the switches $S_{2}$ and $S_{2 C}$ in Fig. 1(b) commutate complementarily at $f_{s w}$ over the entire cycle of the sinusoidal output voltage, whereas the control of the other devices depends on the sign of $v_{\text {grid }}$. During the positive half-wave of the output voltage $S_{1}$ and $S_{1 C}$ commutate complementarily, $S_{3}$ is controlled synchronously with $S_{1}$, and $S_{3 C}$ is always OFF. The gate signals of $S_{1}$ and $S_{2}$ are obtained comparing the same modulation index with two different triangular carriers, phase shifted of $T_{s w} / 2$. Therefore, 4 transitions between positive (negative) and zero states are obtained during a single switching period. When the converter output voltage is 0 two paths are possible for the output current: through $S_{1 C}$ and $S_{2}$ (state $0_{1}^{+}$), or through $S_{2 C}$ and $S_{3}$, called state $0_{2}^{+}$. The behaviour of the system during the negative half-wave is dual. The average device switching frequency is higher than in the previous case. Nevertheless, since some commutations happen in zero-voltage or zero-current conditions, $f_{a v}$ can be effectively considered 


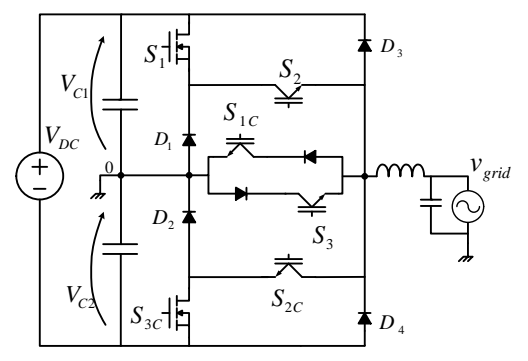

Fig. 3. Topology proposed in [15].

equal to $f_{s w} / 2$ [14].

The same modulation can be used for SNPC and ASNPC converters (Fig. 2). In such cases, due to the presence of two additional devices with respect to ANPC, more converter states and configurations are possible. A solution specifically designed for PV grid-tied applications was proposed in [15] (Fig. 3 ). Employing CoolMOSes and IGBTs, the architecture differs from a traditional SNPC in order to avoid the conduction of the IGBTs antiparallel body diode. Only IGBTs without body diode are used, thus 4 external diodes are added with respect to Fig. 2(a), for guaranteeing the load current free-wheeling paths. Moreover, CoolMOSes are employed for the outer devices, $S_{1}$ and $S_{3 C}$ in Fig. 3, in order to achieve higher efficiency at low output power.

During the zero state the output current is divided into two parallel paths, each with a diode and an IGBT in series, thus reducing the conduction losses. The same basic concept is adopted in this paper, but the reduction of power losses is further improved by means of converter structure simplifications and the use of SiC MOSFETs.

The use of MOSFETs is particularly appealing for PV applications, since the converter efficiency is evaluated not only at the nominal converter power, but over a wide range of different load conditions. In fact, the production of energy from renewable sources is expected to be highly variable and weighted efficiency indexes, such as CEC Efficiency and EU Efficiency, that evaluate the performance of the converter at different percentage of its nominal power, are preferred. In this context, the use of MOSFETs instead of IGBTs allows to achieve high efficiency even at low output power levels, thus improving the overall converter performance. Nevertheless, due to the poor performances of MOSFET body diodes, the solutions proposed in literature adopt techniques to avoid the conduction of MOSFET body diodes, adding devices and complexity to the converter structure [18], [19]. The conduction of the body diode has proven to be not only a source of losses, but also a serious reliability issue [20]. The use of SiC MOSFETs can overcome this issue. The reverse recovery performance of $\mathrm{SiC}$ MOSFET body diodes is much better if compared with their Si counterparts. The modulation proposed in this paper relies on a full-SiC MOSFET ANPC structure to achieve high converter efficiency and higher European efficiency with respect to the solutions proposed so far for ANPC converters.

The proposed ANPC full-SiC MOSFET converter is presented in Fig. 4 along with the driving signals of the power

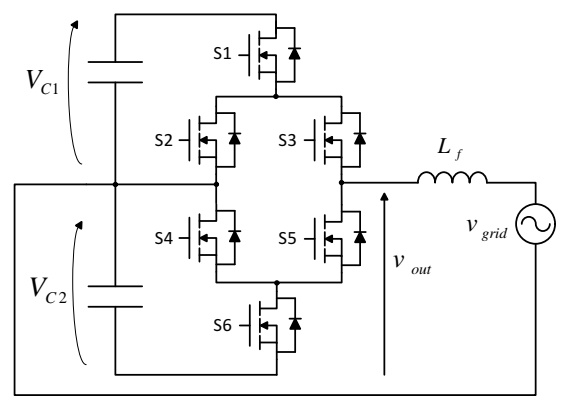

(a)

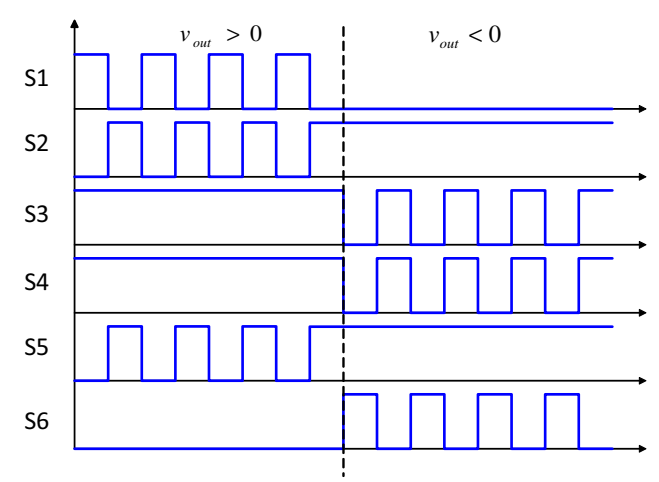

(b)

Fig. 4. ANPC Topology (a) and PWM strategy (b).

devices.

Depending on the driving signals, the possible operating states of the converter are four and are depicted in Fig. 5. During the positive half-wave of the desired output voltage, when $S_{1}$ and $S_{3}$ are $\mathrm{ON}$, the converter feeds positive voltage to the output (active-state), state $P$ in Fig. 5. The current flows through two devices in series. $S_{4}$ is ON, but it is not conducting current. When $S_{1}$ switches off, $S_{5}$ and $S_{2}$ switch on simultaneously, thus the current is divided in two paths in parallel: $S_{2}-S_{3}$, and $S_{4}-S_{5}$ (state $0^{+}$in Fig. 5). The voltage at the output of the converter is zero (zero-state). Similarly, when the converter feeds negative voltage, $S_{5}$ and $S_{6}$ are ON (state $N)$. Since $S_{2}$ is also ON, the $v_{d s}$ voltages for $S_{1}$ and $S_{3}$ are equal to $V_{D C} / 2$. When $S_{6}$ is switched off the whole H-bridge composed by $S_{2}-S_{3}-S_{4}-S_{5}$ acts as a conduction path (state $\left.0^{-}\right)$. As MOSFETs exhibit good current sharing capability, this method can ensure low conduction losses.

The fact that three devices are commutated during a switching period does not imply an increase of switching losses. As a matter of fact two devices are kept ON during the whole grid voltage half-period (e.g. $S_{3}$ and $S_{4}$ during the positive half-wave of the output voltage), while the other two ( $S_{2}$ and $S_{5}$ under the same hypothesis) are gated under zero voltage switching (ZVS) conditions. Therefore this PWM strategy theoretically implies very low switching losses $\left(f_{a v}=f_{s w} / 2\right)$ and low conduction losses as well. As in all NPC derived structures, the drawback resides in the fact that the DC link voltage must be greater than twice the grid voltage peak. As a consequence, comparatively high switching frequencies or 


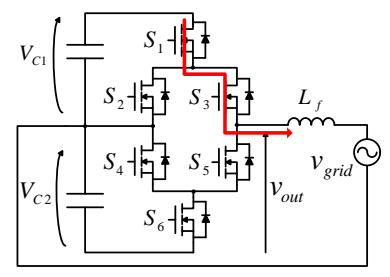

$(\mathrm{P})$

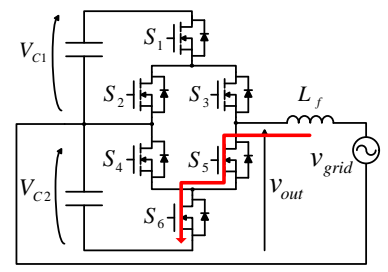

(N)

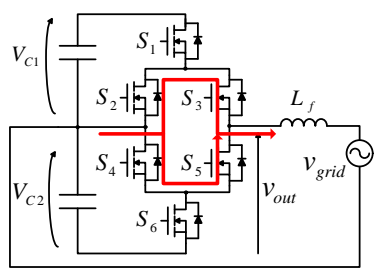

$\left(0^{+}\right)$

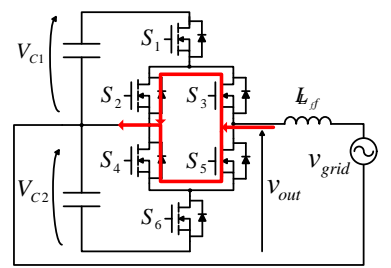

$\left(0^{-}\right)$
Fig. 5. Converter states.

TABLE II. SUMMARY OF THE CONVERTER'S CHARACTERISTICS

\begin{tabular}{|c|c|c|c|}
\hline Converter topology & $f_{a v}$ & $f_{a p}$ & number of devices \\
\hline NPC & $f_{s w} / 2$ & $f_{s w}$ & 4 IGBTs + 2 Diodes \\
\hline ANPC in [11] & $f_{s w} / 2$ & $2 f_{s w}$ & 6 IGBTs \\
\hline SNPC in [15] & $f_{s w} / 2$ & $f_{s w}$ & $\begin{array}{c}\text { 2 CoolMOSs + 4 IGBTs } \\
+6 \text { Diodes }\end{array}$ \\
\hline $\begin{array}{c}\text { Full-SiC MOSFET converter } \\
\text { with proposed modulation }\end{array}$ & $f_{s w} / 2$ & $f_{s w}$ & 6 MOSFETs \\
\hline
\end{tabular}

bulky output filter inductors must be employed to limit the output current ripple with respect to a full-bridge based PV inverter. Again, the use of SiC MOSFETs for this architecture allows to operate at high switching frequencies while keeping a fairly small inductive filter.

Moreover, it is important to note that the maximum voltage across the devices during converter operation is $V_{D C} / 2$; therefore $650 \mathrm{~V} \mathrm{SiC} \mathrm{MOSFETs} \mathrm{can} \mathrm{be} \mathrm{used,} \mathrm{since} \mathrm{a} \mathrm{minimum} \mathrm{of}$ $700 \mathrm{~V}$ for the DC-link voltage is required for injecting current into the grid.

The characteristics of the Full-SiC-MOSFET converter with the proposed modulation technique are resumed in TABLE II, along with characteristics of the converters taken as benchmarks for this work.

\section{TheOretical POWER Loss ANALYSIS}

Since the efficiency of PV transformerless inverters is normally assessed using weighted efficiency measures such as CEC Efficiency and EU Efficiency, it is critical to evaluate power semiconductor device losses at different load conditions rather than at nominal load condition.

The device power losses can be divided in conduction and switching losses. The voltage drop across the device during a conduction stage can be expressed as in (1) [4]:

$$
v_{\text {con }}=\left\{\begin{array}{l}
\text { MOS }: v_{d s}=i(t) R_{d s} \\
I G B T: v_{c e}=V_{t}+i(t) R_{c e} \\
\text { Diode }: v_{a k}=V_{f}+i(t) R_{a k}
\end{array}\right.
$$

where $v_{d s}$ is the MOSFET drain-source voltage drop, $R_{d s}$ is the MOSFET drain-source on resistance, $v_{c e}$ is the IGBT collector-emitter voltage drop, $V_{t}$ is the IGBT equivalent voltage drop under zero current condition, $R_{c e}$ is the IGBT on resistance, $v_{a k}$ is the diode anode-cathode voltage drop, $V_{f}$ is the diode equivalent voltage drop under zero current condition, $R_{a k}$ is the diode on resistance, and $i(t)$ is the current through the device.

Considering only the positive half-wave of the output voltage active-state, the power losses over a single device are given by (2), where $i(t)=I_{m} \sin (\omega t+\theta)$, with $I_{m}$ as the peak of the inverter output current, $\omega$ its angular frequency and $\theta$ the phase displacement between grid current and voltage. $D_{\text {active }}$ is the duty ratio for the active stage. For a grid-connected inverter the duty ratio for active state and zero-state can be expressed as (3), where $M$ has a value comprised between 0 and 1. Combining (2) with (3) and (1), the conduction losses for a single device during the active state can be expressed as (4) for MOSFETs and IGBTs.

Conversely, the conduction power loss for a single device during zero-state is given in (5), where $i_{z e r o}(t)$ is used to indicate the current flowing through the device. $i_{z e r o}(t)$ is used for generality since, according to the adopted modulation strategy, $i_{z e r o}(t)$ can be equal to $i(t)$ or $i(t) / 2$. Finally, in (6) the zero-state conduction losses are given for MOSFET, IGBT and diode cases, with $I_{m_{-} \text {zero }}$ as the peak of $i_{\text {zero }}(t)\left(I_{m}\right.$ or $I_{m} / 2$ depending on the modulation strategy).

$$
\begin{aligned}
& P_{\text {con_act }}=\frac{1}{2 \pi} \int_{0}^{\pi} v_{\text {con }} i(t) D_{\text {active }}(t) \mathrm{d}(\omega t) \\
& D_{\text {active }}(t)=M \sin (\omega t) \\
& D_{\text {zero }}(t)=1-M \sin (\omega t) \\
& P_{\text {con_act }}=\left\{\begin{aligned}
\text { MOS }: & \frac{I_{m}^{2} R_{d s} M}{2 \pi}\left(1+\frac{1}{3} \cos (2 \theta)\right) \\
I G B T: & I_{m} V t \frac{M}{4} \cos (\theta) \\
& +\frac{I_{m}^{2} R_{c e} M}{2 \pi}\left(1+\frac{1}{3} \cos (2 \theta)\right)
\end{aligned}\right. \\
& P_{\text {con_zero }}=\frac{1}{2 \pi} \int_{0}^{\pi} v_{\text {con }} i_{z e r o}(t) D_{z e r o}(t) \mathrm{d} \omega t
\end{aligned}
$$

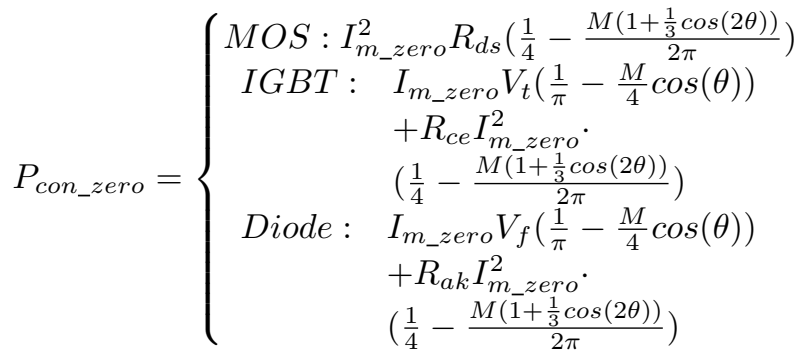

For the switching losses, data sheets usually report the turn-on/turn-off energy losses of the devices, measured under precise test conditions at different junction temperatures and for given values of the switching voltage and current $\left(V_{\text {test }}, I_{\text {test }}\right)$. In particular, for MOSFET devices, the main loss source for switching transitions is the capacitive turnon energy loss $\left(E_{\text {oss }}\right)$ resulting from the discharge of the 


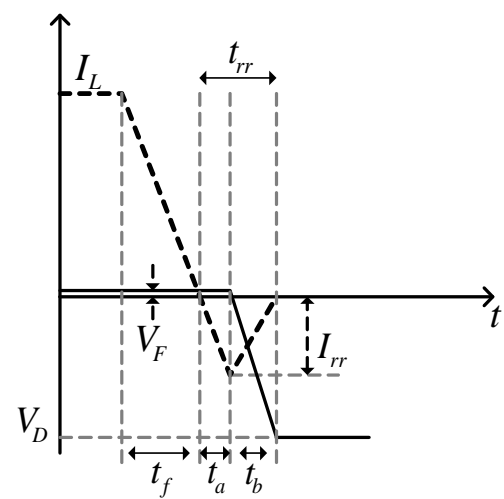

Fig. 6. Simplified diode switching waveforms during turn-off. Voltage (solid line), current (dashed).

junction capacitor $C_{\text {oss }}$ of MOSFETs, which is dependent on the switched drain-source voltage across the device before the switch-on transition. Moreover, it is important to note that, in the case of the converters under exam, the switching voltage to consider for the calculations is $V_{D C} / 2$, since the commutations happen with half the DC-link voltage. Therefore, the switching losses for a single device (MOSFET or IGBT) during a period of $v_{\text {grid }}$ can be expressed as in (7), considering $E_{\text {on }}$ and $E_{\text {off }}$ from the data sheets, and normalizing them to the switching conditions considered for the measurements.

Additional switching losses in the outer devices of the ANPC/NPC structure are caused by the reverse recovery current of the inner diodes. The extra turn-on energy loss can be expressed as in (8), where $t_{a}, t_{b}$ and $I_{r r}$ are defined in Fig. 6 , and $I_{L}$ is the switched load current. Considering a sinusoidal current, the extra power losses for one outer device over a grid period are given in (9).

Conversely, the switch-off losses for the diode can be expressed as in (10), whereas the turn-on losses are neglected.

$$
\begin{gathered}
P_{O N}=\left(\frac{I_{m}}{\pi} \frac{V_{D C}}{2}\right) f_{s w} \frac{E_{o n}}{V_{\text {test }} I_{\text {test }}} \\
P_{O F F}=\left(\frac{I_{m}}{\pi} \frac{V_{D C}}{2}\right) f_{\text {sw }} \frac{E_{\text {off }}}{V_{\text {test }} I_{\text {test }}} \\
E_{\text {extra_ON }}=\frac{V_{D C}}{2}\left(I_{L}+\frac{I_{r r}}{2}\right) t_{a}+\frac{V_{D C}}{2} \frac{I_{r r}}{3} t_{b} \\
P_{\text {extra_ON }}=\frac{1}{2 \pi} \int_{0}^{\pi} E_{\text {extra_ON }} f_{s w} \mathrm{~d} \omega t \\
=\frac{V_{D C}}{4} f_{s w}\left(\left(\frac{2 I_{m}}{\pi}+\frac{I_{r r}}{2}\right) t_{a}+\frac{I_{r r}}{3} t_{b}\right) \\
P_{\text {Diode_OF }}=\frac{1}{2 \pi} \int_{0}^{\pi} \frac{1}{6} t_{b} I_{R R} \frac{V_{D C}}{2} f_{s w} \mathrm{~d} \omega t \\
=\frac{1}{12} t_{b} I_{R R M} \frac{V_{D C}}{2} f_{s w}
\end{gathered}
$$

\section{A. Power Losses in NPC converters}

During the active states two IGBTs in series conduct the current, whereas the current passes through one diode and one IGBT during zero states $\left(I_{m_{-} z e r o}=I_{m}\right.$ in (6)). The outer switches $S_{1}, S_{2 C}$ commutate in hard switching conditions. Moreover, when they turn on, additional losses, due to the reverse recovery currents of $D_{1}$ and $D_{2}$ respectively, are present. The turn-off losses during a passage from zero to an active state have to be considered, but the turn-on losses at the beginning of a zero state can be neglected, as well as the low frequency switching losses of the inner IGBTs. All the above considerations yield the total power loss over a grid voltage period, as given in (11).

$$
\begin{aligned}
P_{\text {con_act }}= & 2\left(2 P_{\text {con_act }}(I G B T)\right) \\
P_{\text {con_zero }}= & 2\left(P_{\text {con_zero }}(\text { Diode })+P_{\text {con_zero }}(I G B T)\right) \\
P_{\text {sw }}= & 2\left(P_{O N}+P_{O F F}\right. \\
& \left.+P_{\text {Diode_OFF }}+P_{\text {extra_ON }}\right)
\end{aligned}
$$

\section{B. Power Losses in ANPC}

With the modulation strategy proposed in [11], during a switching period, 2 active states and 2 zero states are present. Considering the positive half-wave of the desired output voltage, the succession of states $0_{1}^{+}, P, 0_{2}^{+}, P$ is repeated at the switching frequency. The zero-states differ because the path for the output current is different, but in both cases one IGBT and one IGBT antiparallel diode in series carry the current. During state $P$ two IGBTs in series are ON, therefore the total conduction losses are equal to the previous case of NPC converter.

Analyzing the switching losses, it can be noted that during a transition from $0_{1}^{+}$to $P$, the reverse recovery losses of the antiparallel diode of $S_{1 C}$ are added to the switch-on losses of $S_{1}$ (Fig. 1b), whereas $S_{3}$ turns on under zero current switching (ZCS) conditions. When the transition from $P$ to $0_{2}^{+}$happens, $S_{2}$ commutates in hard switching, while $S_{2 C}$ in ZVS as its antiparallel diode turns on before the device is gated. In the subsequent passage from $0_{2}^{+}$to $P$, the reverse recovery of the antiparallel diode of $S_{2 C}$ must be considered in the calculation of the switch-on losses of $S_{2}$. Finally, in the commutation between $P$ and $0_{1}^{+}$, only the switch-off losses of $S_{1}$ are taken into account since the turn-on of the $S_{1 C}$ antiparallel diode is considered ideal.

Therefore, it is possible to state that the total switching losses are doubled with respect to the case of NPC converter. Nevertheless, the apparent switching frequency of the output voltage is twice the previous case, and the losses are evenly distributed among all devices. The ANPC power losses are summarized in (12). 


$$
\begin{aligned}
P_{\text {con_act }}= & 2\left(2 P_{\text {con_act }}(I G B T)\right) \\
P_{\text {con_zero }}= & 2\left(P_{\text {con_zero }}(\text { Diode })+P_{\text {con_zero }}(I G B T)\right) \\
P_{\text {sw }}= & 2 \times 2\left(P_{O N}+P_{O F F}\right. \\
& \left.+P_{\text {Diode_OFF }}+P_{\text {extra_ON }}\right)
\end{aligned}
$$

\section{Power Losses in SNPC converters}

For the converter proposed in [15], during active states two devices, a CoolMOS and an IGBT, are ON. During the zero state the current flows in two parallel paths, each containing a diode and an IGBT; therefore in (5), $i_{\text {zero }}(t)$ is equal to $i(t) / 2$ and $I_{m \_z e r o}=I_{m} / 2$.

For the switching losses, particular attention must be paid to the reverse recovery behaviour of the diodes. In this case, during a transition from zero to active states, two diodes switch off instead of only one as in the previous cases. Nevertheless, it has to be considered that the reverse recovery current amplitude $\left(I_{r r}\right)$ depends on the amplitude of the current that is passing through the device before the switching transition; since each device conducts half of the total load current, it is possible to approximate the switching losses related to the diodes' commutations as equal to the previous cases. Furthermore, considering the presence of a CoolMOS as outer device, the total losses are given in (13)

$$
\begin{aligned}
P_{\text {con_act }}= & 2\left(P_{\text {con_act }}(I G B T)+P_{\text {con_act }}(M O S)\right) \\
P_{\text {con_zero }}= & 2 \times 2\left(P_{\text {con_zero }}(\text { Diode })+P_{\text {con_zero }}(I G B T)\right) \\
P_{\text {sw }}= & 2\left(P_{\text {ON }}+P_{O F F}\right. \\
& \left.+P_{\text {Diode_OFF }}+P_{\text {extra_ON }}\right)
\end{aligned}
$$

\section{Power Losses with the Proposed Modulation}

During the active states two devices are on, as before. During the zero-state the devices $S_{2}, S_{3}, S_{4}, S_{5}$ are ON, the current is equally divided in two parallel paths: through the series $S_{2}-S_{3}$, and the series $S_{4}-S_{5}\left(I_{m_{-} \text {zero }}=I_{m} / 2\right)$. Therefore, it is possible to account the conduction power losses as the current would flow through a single device with on-state resistance equal to $R_{d s}$. Furthermore, as a dead time is inserted in the commutation signals, there is a short interval in which the current is flowing through the MOSFET antiparallel diode, causing additional losses. Considering a dead time $t_{d t}$ of 250 ns the additional losses for a half period of the grid voltage are expressed in (14).

$$
\begin{aligned}
P_{\text {con_dt }}= & \left(I_{m_{-} z e r o} V_{f}\left(\frac{1}{\pi}-\frac{M}{4}\right)\right. \\
& \left.+I_{m \_z e r o}^{2} R_{a k}\left(\frac{1}{4}-\frac{2 M}{3 \pi}\right)\right) t_{d t} f_{s w}
\end{aligned}
$$

For the switching losses, considerations are similar to the SNPC converter. The main difference in this case resides in the use of $\mathrm{SiC}$ devices, that have a less marked reverse recovery

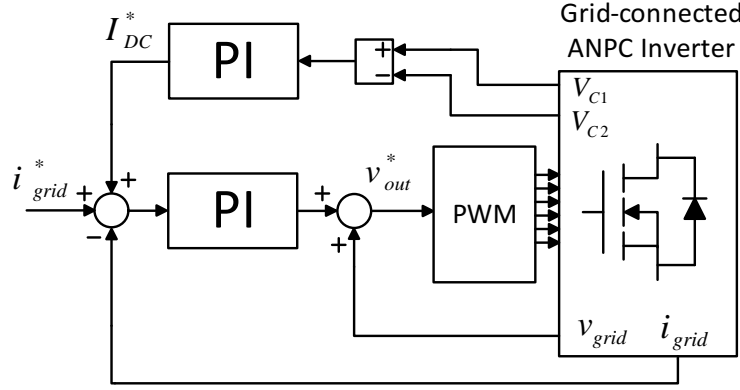

Fig. 7. Block scheme of the current control.

behavior with respect to traditional $\mathrm{Si}$ devices; therefore the total switching losses are expected to be very low.

The total losses are resumed in (15).

$$
\begin{aligned}
P_{\text {con_act }}= & 2\left(2 P_{\text {con_act }}(M O S)\right) \\
P_{\text {con_zero }}= & 2 \times 2\left(2 P_{\text {con_zero }}(M O S)+P_{\text {con_dt }}\right) \\
P_{s w}= & 2\left(P_{O N}+P_{O F F}\right. \\
& \left.+P_{\text {Diode_OFF }}+P_{\text {extra_ON }}\right)
\end{aligned}
$$

\section{Simulation Results and Power Loss Calculations}

The proposed modulation was simulated in the MATLAB/Simulink environment with the PLECS toolbox. The simulation circuit reflects the schematic of Fig. 4(a), with $L_{f}=1 \mathrm{mH}$, the equivalent capacitance of one half of the DC link $C=2 \mathrm{mF}$, and considering $800 \mathrm{~V}$ for the total DC link voltage.

The control of the converter is shown in Fig. 7, where a simple Proportional-Integral (PI) regulator is used to control the injected grid current. The grid voltage is added after the regulator as a feed-forward term. The balancing of the DC link capacitor is realized with a PI regulator that control the DC component of the grid current. If the injected grid current has no DC component and the capacitor voltages are balanced, the power drawn from each capacitor is equal. This also ensures that no DC current is injected into the grid. This aspect is of paramount importance, because the DC current injection from grid-connected converters must respect strict limits imposed by international regulations.

Fig. 8 shows the grid voltage and current when $3 \mathrm{~kW}$ of active power is delivered to the grid, with a switching frequency $f_{s w}=40 \mathrm{kHz}$.

In Fig. 8 the output voltage does not have flat tops because the capacitor voltages are changing within the grid voltage period. As a matter of fact, in unity power factor condition, the output is connected to the upper half of the DC link during the positive half-wave of the grid voltage, and to the lower half otherwise. This causes a voltage change over the $10 \mathrm{~ms}$ of the half wave, which is visible in the simulations.

The total losses of a full-SiC-MOSFET converter, that features the proposed modulation strategy, were calculated 

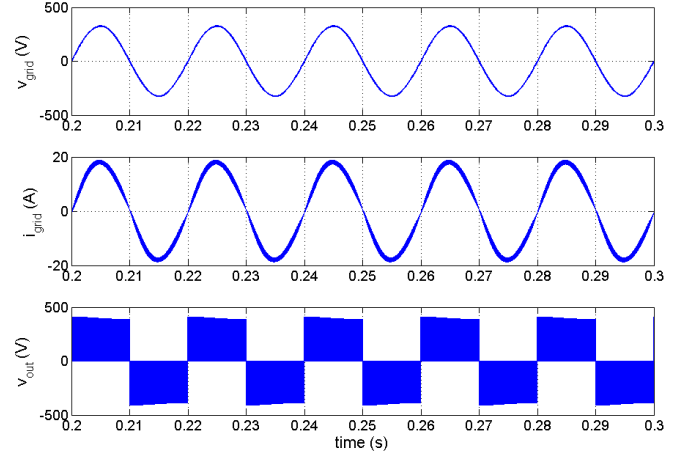

Fig. 8. Simulation results.

TABLE III. DEVICE PARAMETERS AT $25^{\circ} \mathrm{C}$

\begin{tabular}{|c|c|c|c|c|c|c|c|}
\hline Device & $\begin{array}{c}R_{d s} l \\
R_{c e} \\
m \Omega\end{array}$ & $V_{t}$ & $V_{f}$ & $R_{a k}$ & $I_{r r}$ & $t_{a}$ & $t_{b}$ \\
\hline IGBT & 15 & 1.7 & 0.75 & 75 & 3 & 29.4 & 20.6 \\
STGW35HF60WDB & & & & & & & \\
\hline $\begin{array}{c}\text { SiC MOSFET } \\
\text { SCT2120AF }\end{array}$ & 120 & - & 1.4 & 290 & 3 & 17 & 16 \\
\hline $\begin{array}{c}\text { CoolMos } \\
\text { IPW60R045CP }\end{array}$ & 45 & - & - & - & - & - & - \\
\hline $\begin{array}{c}\text { Diode } \\
\text { FFP30S60S }\end{array}$ & - & - & 0.8 & 80 & 2.4 & 14 & 14 \\
\hline
\end{tabular}

according to the formulae in Section III, for different values of delivered output power. The power losses were evaluated considering the parameters of TABLE III and compared with those of NPC, ANPC and SNPC converters.

Moreover, in order to highlight the advantages of employing MOSFETs instead of IGBTs, the power losses using the proposed modulation with an ANPC converter adopting IGBTs as the main switches, were calculated as well.

CoolMOS IPW60R045CP and soft switching diodes FFP30S60S were considered for the SNPC topology, as indicated in [15]. The adopted IGBTs are STGW35HF60WDB by STMicroelectronics, and the performance of their antiparallel body diode was also used for the single diodes of the NPC structure. The SiC MOSFETs were $650 \mathrm{~V}$ SCT2120AF by ROHM Semiconductor.

In Fig. 9 the power losses calculated for the converters under exam are displayed at different percentages of the nominal converter power of $3 \mathrm{~kW}$. In particular, it can be observed that the full-SiC MOSFET ANPC converter with the proposed modulation strategy exhibits the highest efficiency at 10, 30, 50 percent of the nominal power, due to the better performance of MOSFETs at light load. At full power the SNPC, thanks to the very low on-state resistance of CoolMOSes, has almost the same efficiency as the proposed solution. Nevertheless, the performance of the proposed modulation remains higher than those of the other converters, but the benefits in employing MOSFETs instead of IGBTs are lower, because at $3 \mathrm{~kW}$ the performances of the chosen IGBTs are similar to those of the SCT2120AF SiC MOSFETs.

Moreover the zero-state conduction losses, thanks to the proposed modulation technique that enables the parallel op-

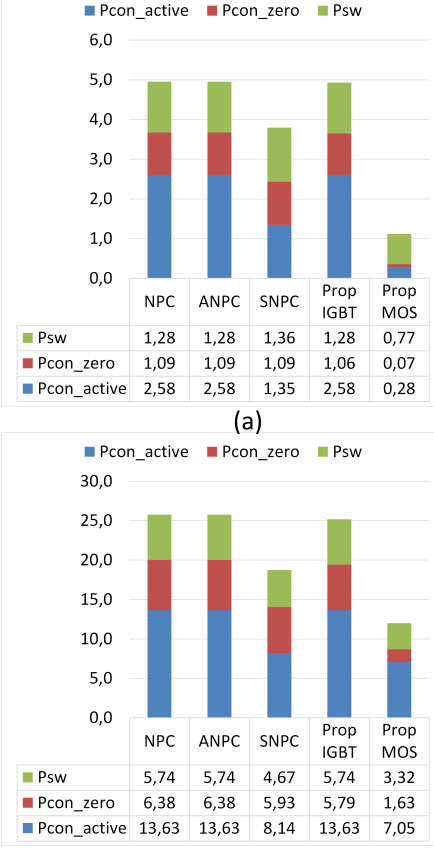

(c)

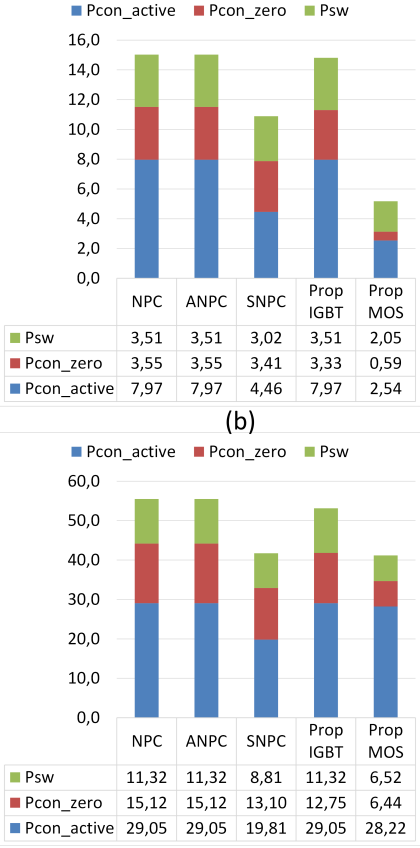

(d)
Fig. 9. Theoretical power losses of NPC, ANPC, SNPC and the proposed modulation with IGBT or $\mathrm{SiC}$ devices at $10 \%$ (a), 30\%(b), 50\%(c) and $100 \%$ (d) of the $3 \mathrm{~kW}$ nominal power.

TABLE IV. EUROPEAN AND CEC EFFICIENCY

\begin{tabular}{|c|c|c|}
\hline Converter topology & European Efficiency & CEC Efficiency \\
\hline NPC & $98.3 \%$ & $98.2 \%$ \\
\hline ANPC in [11] & $98.3 \%$ & $98.2 \%$ \\
\hline SNPC in [15] & $98.7 \%$ & $98.7 \%$ \\
\hline Prop. Modulation with IGBT & $98.3 \%$ & $98.3 \%$ \\
\hline Prop. Modulation with SiC & $99.1 \%$ & $99 \%$ \\
\hline
\end{tabular}

eration of the MOSFETs, are the less significant in any case. Further benefits to the efficiency can be expected when the converter operates under non-unity power factor conditions. The switching losses are very low as can be expected with $\mathrm{SiC}$ devices, thus it can be stated that the total efficiency of the converter will not be highly dependent on the switching frequency in the range of considered switching frequencies $(10-40 \mathrm{kHz})$.

The ratio between the conduction active-state and zero-state losses depends on the ratio between the DC link voltage and the grid, since, for a given value of desired output current, the duration of the freewheeling state (zero-state) is larger when the DC source voltage is higher.

The calculated European efficiency and CEC efficiency for the converters under exam are reported in TABLE IV.As can be seen, the full-SiC MOSFET ANPC with the proposed modulation exhibits the highest efficiencies thanks to the better performance at low power.

\section{A. Discussion on the power losses of the different topologies}

As can be seen from Fig. 9, the NPC and ANPC have the same losses, if the comparison is done with the same output 


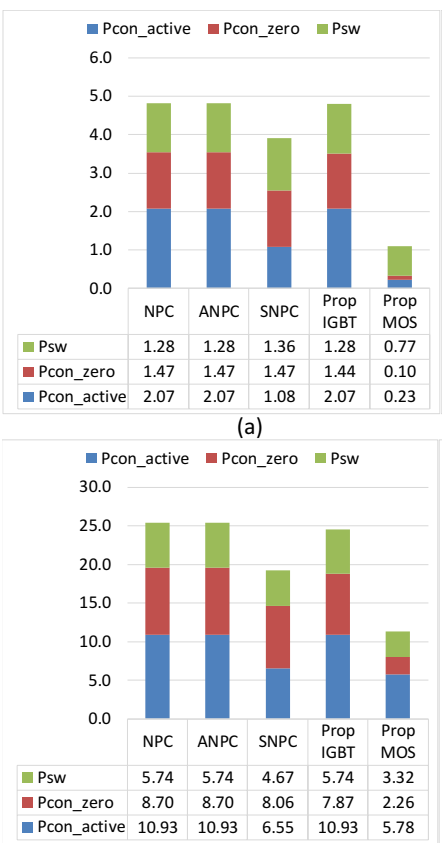

(c)

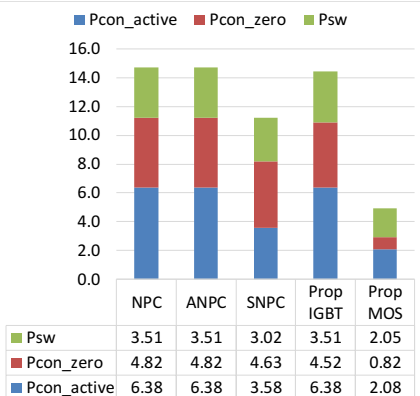

(b)

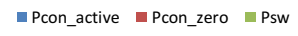

60.0

50.0

40.0

30.0

20.0

10.0

0.0 NPC ANPC SNPC Prop Prop \begin{tabular}{|l|c|c|c|c|c|}
\hline $\mathbf{M}$ Psw & 11.32 & 11.32 & 8.81 & 11.32 & 6.52 \\
\hline $\mathbf{M}$ Pcon_zero & 20.70 & 20.70 & 17.86 & 17.38 & 8.97 \\
\hline
\end{tabular} \begin{tabular}{|l|l|l|l|l|l} 
acon_active & 23.31 & 23.31 & 15.99 & 23.31 & 23.14
\end{tabular}

(d)

Fig. 10. Theoretical power losses of NPC, ANPC, SNPC and the proposed modulation with IGBT or SiC devices at $10 \%(\mathrm{a}), 30 \%(\mathrm{~b}), 50 \%$ (c) and $100 \%$ (d) of the rated full current and power factor $=0.8$.

THD. The benefits of the proposed PWM when Si devices are used are not evident, due to the threshold behavior of the bipolar devices, that show a constant component of the on-state voltage drop even at low current levels. The SNPC benefits from the adoption of the CoolMOSes, with a more complex architecture. However, if the operation is constrained to unity power factor, the adoption of CoolMOSes for high and low side devices is feasible even for the standard NPC.

What is important to highlight is that the adoption of $\mathrm{SiC}$ MOSFETs and the proposed PWM allows to greatly reduce the conduction losses during the zero state, even considering devices with a smaller package (TO220 against TO247).

\section{B. Benefits of the modified PWM with non-unity power factor operation}

References (2) and (6) show a dependency of the active and zero states conduction power losses to the phase displacement $\theta$ between grid current and voltage. In particular, regardless of the considered converter topology, as the power factor decreases the zero state conduction losses increase whereas those in active states decrease. This because there are more zero states facing a higher value of grid current relative to the case of unity power factor. Since grid connected converters have been recently requested to inject into the grid also a certain amount of reactive power, the converter efficiency in case of non-unity power factor must be taken into account to correctly evaluate the converter performance.

Fig. 10 reports the calculated losses for the converters under examination in case of $P F=0.8$ and the same current
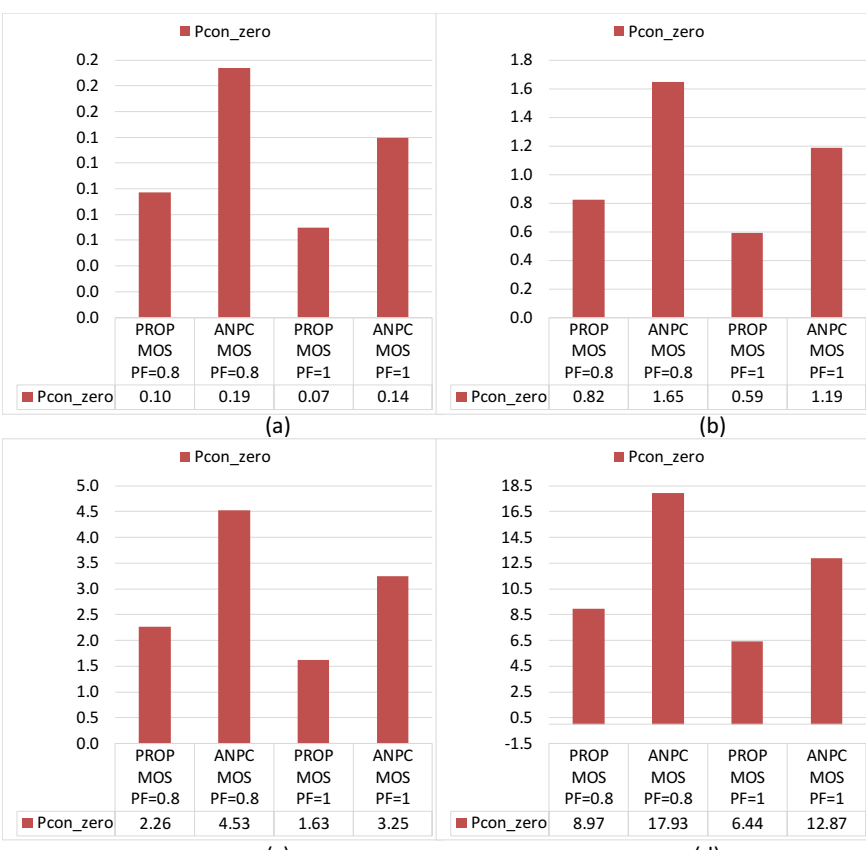

(c)

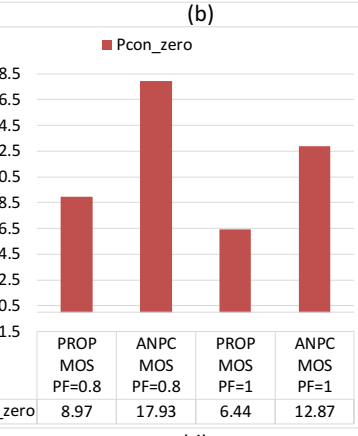

(d)

Fig. 11. Comparison between ANPC with SiC and ANPC with SiC and proposed modulation at $10 \%(\mathrm{a}), 30 \%(\mathrm{~b}), 50 \%(\mathrm{c})$ and $100 \%(\mathrm{~d})$ of the rated full current and different power factors.

amplitude considered for the data in Fig. 9. As can be seen, the ANPC coverter with SiC MOSFET and the proposed modulation is the one that performs better. In this case the impact of the proposed modulation on the converter efficiency is more evident as the conduction losses during zero states counts more relative to the total losses. SNPC maintains the lowest active conduction losses but, in comparison to the full $\mathrm{SiC}$ ANPC with the proposed modulation, pays more losses during zero states, resulting in a lower efficiency.

For the sake of completeness, in Fig. 11 an evaluation of the loss reduction achievable with the proposed modulation against the other ANPC solutions is performed. As can be seen, the losses during the zero state are exactly half, and the advantage is more evident considering non-unity power factors.

\section{EXPERIMENTAL RESULTS}

The experiments were performed with a prototype converter based on the Freescale MC56F8323 MCU. The converter generates the power supplies needed for the logic and the gate driver circuits from the DC link. The DC source used was a DC Power supply that provides $700 \mathrm{~V}$ with up to $2 \mathrm{~kW}$ of continuous power. The converter output was connected to the secondary of an isolation transformer with $230 \mathrm{~V} \mathrm{rms}$ and $50 \mathrm{~Hz}$ at the point of connection. Fig. 12 shows the output current and voltage in case of unity power factor (PF) and $2 \mathrm{~kW}$ of delivered output power for two different values of the switching frequency. Differently from the simulations, the grid voltage shows a marked third harmonic distortion. This behavior is quite common in the low-voltage grids, because 
of the widespread adoption of diode-bridge rectifiers as input stages. This third harmonic distortion causes also a small lowfrequency distortion in the grid current, that could have been compensated with a more complex architecture of the current controller. However, the optimization of the current controller under distorted grid voltage is outside the scope of the paper.

The efficiency measurements were done with a N4L PPA5530 power analyzer, measuring the DC input power and the output power after the filter inductor, excluding the transformer losses.

The performance of the converter was evaluated both with the ROHM Semiconductor SCT2120AF SiC MOSFETs and the STMicroelectronics STGW35HF60WDB silicon IGBTs, for switching frequencies of 10,20 and $40 \mathrm{kHz}$ and different temperatures of the heat sink $(25,50,75,100$ degrees Celsius for the SiC MOSFETs, up to $75 \mathrm{C}$ for the IGBTs to avoid exceeding the maximum rated junction temperature).

In this work, the authors decided to perform the comparison considering the same heatsink temperature, while a fairer comparison would have been achieved considering the same junction temperature. However, the junction temperature is not directly measurable in an easy way, for this reason the heat sink temperature was controlled. This choice has consequences that need a supporting discussion.

As a premise, since different devices carry a different current during the grid voltage period, it is safe to assume that the junction temperatures will have some differences even inside the same test case ( $\mathrm{Si}$ or $\mathrm{SiC}$ ), making a test under the same junction temperature not feasible. The STGW35HF60WDB device presents a thermal resistance junction-case of $0.63 \mathrm{C} / \mathrm{W}$ for the IGBT and $1.5 \mathrm{C} / \mathrm{W}$ for the diode, while the SCT2120AF has $0.7 \mathrm{C} / \mathrm{W}$. Considering that the results were obtained under unity power factor, the diode conduction happens only during the zero state. The zero state is applied for a longer time when the grid current is low, so it can be concluded that the diode losses constitute a small part of the total losses.

As an approximation, it is safe to say that the $\mathrm{Si}$ and $\mathrm{SiC}$ devices have similar thermal impedance, also considering that a very important thermal resistance contribution is given by the thermal pad between the case and the heatsink. Under these assumptions, $\mathrm{Si}$ and $\mathrm{SiC}$ devices will have similar junction temperature when the losses are similar. Since an on-line control of the junction temperature or of the losses is not feasible, if the trials are done under the same heat-sink temperature, the $\mathrm{SiC}$ and $\mathrm{Si}$ devices will have similar expected junction temperatures at $1.5 \mathrm{~kW}$, while the $\mathrm{SiC}$ will benefit from the lower losses in the low power range.

A flyback converter directly connected to the DC link is used to generate the auxiliary power supplies, and these losses are included in the input power. The magnitude of these additional losses was measured to be $12.5 \mathrm{~W}$ when the converter is operating at $10 \mathrm{kHz}$. Considering that the auxiliary power supply losses and the output filter are kept the same for both IGBT and $\mathrm{SiC}$ converters, this has an impact on the maximum efficiency. For this reason, the measures must be read comparing the gain that can be achieved by employing $\mathrm{SiC}$ devices.

Differently from the theoretical analysis of Fig. 9, the

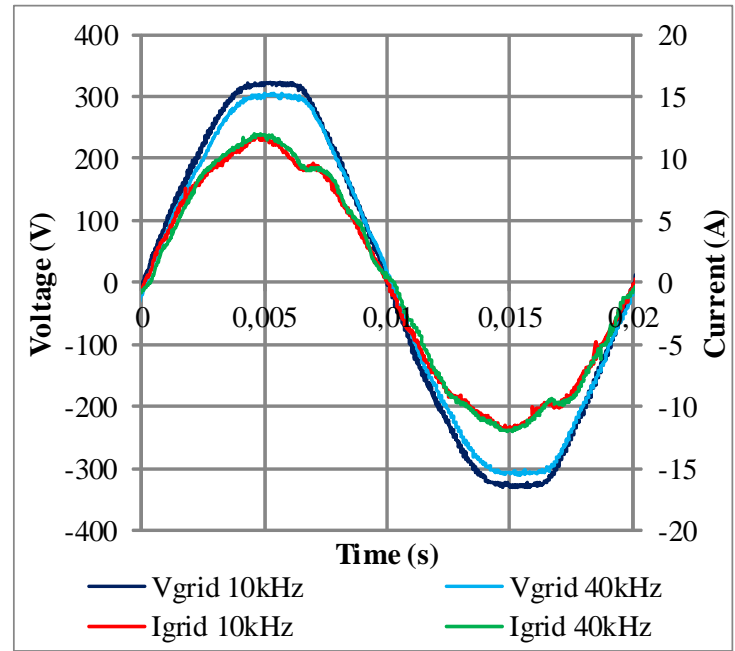

Fig. 12. Grid voltage and current in case of $2 \mathrm{~kW}$ of delivered output power with $f_{s w}$ equal to 10 and $40 \mathrm{kHz}$.

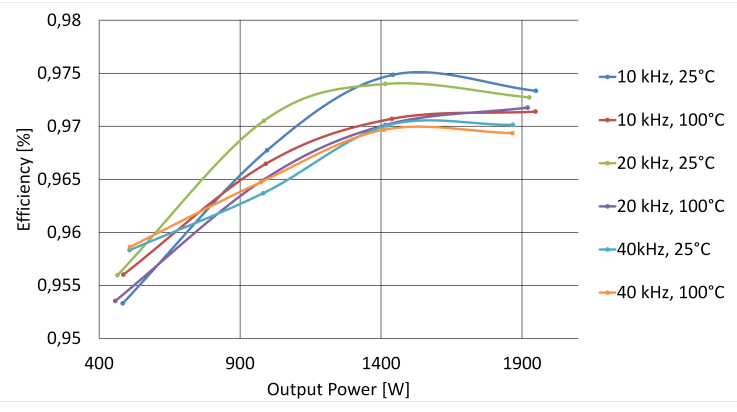

Fig. 13. Total efficiency of the ANPC converter with SiC MOSFETs as a function of switching frequency and operating temperature.

losses on the converter output filter and auxiliary supply were included in the experimental measurements. Furthermore, the maximum converter output power was limited to $2 \mathrm{~kW}$ due to the DC power supply.

Fig. 13 reports the measured efficiency curves for the ANPC converter with SiC MOSFETs, while Fig. 14 refers to the

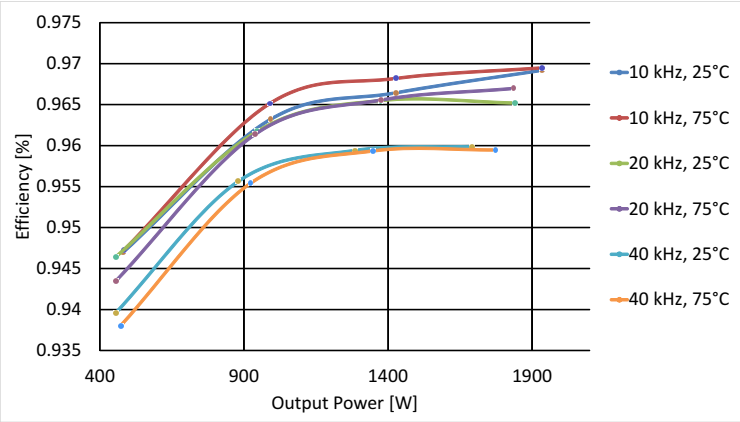

Fig. 14. Total efficiency of the ANCP converter with silicon IGBTs as a function of switching frequency and operating temperature. 


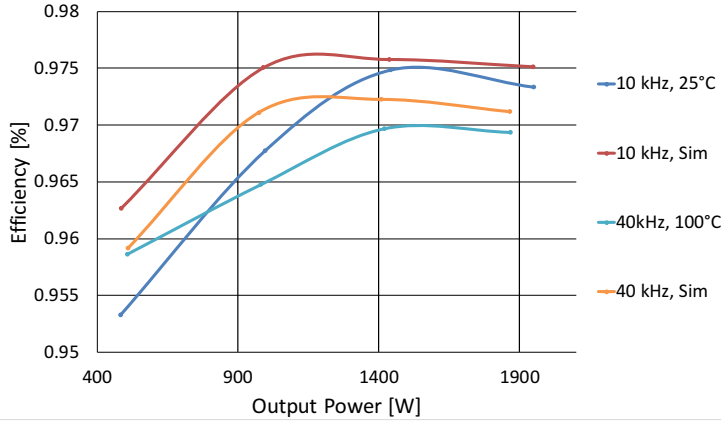

Fig. 15. Comparison between simulated and experimental efficiency of the ANCP converter with SiC MOSFETs.

use of silicon IGBTs. The advantage of the SiC MOSFETs is evident, especially at fractional power and at high temperature. The $\mathrm{SiC}$ MOSFETs maintain better performances than the IGBTs even though they come in a TO-220 package, smaller than the TO-247 of the IGBTs. Using SiC MOSFETs, the switching frequency can be doubled achieving roughly the same efficiency as with silicon IGBTs. This result is in good agreement with similar measurements presented in literature [21].

Finally, Fig. 15 reports a comparison between the simulated total efficiency of the full SiC ANPC converter, taking into account also the simulated losses in the filter inductors (copper and iron losses) and the logic losses, and the total efficiency measured throughout the experimental results. As can be seen, the simulated efficiency is slightly higher than the measured one. This can be due to the mismatch between the junction and heatsink temperatures and to other unmodeled loss sources, i.e., the capacitors ESR.

\section{CONCLUSION}

In this paper an improved modulation for an active NPC inverter equipped with $650 \mathrm{~V} \mathrm{SiC} \mathrm{MOSFETs} \mathrm{was} \mathrm{proposed.}$ The proposed modulation reduces conduction power losses thanks to the parallel operation of the MOSFETs during the free-wheeling intervals. A theoretical analysis of the power losses for different percentages of the nominal output power of the converter was presented in order to evaluate the European efficiency. Moreover, a performance comparison with NPC, ANPC, SNPC inverters was included. For evaluating the actual advantages of using SiC MOSFETs, a comparison with the performance obtained by the same modulation driving an ANPC converter with IGBTs was provided as well. The proposed modulation was proven to yield the greatest benefits if adopted to drive MOSFETs. Furthermore, the SiC MOSFETs maintain better performance at higher frequencies and temperatures, even though they come in a TO-220 package smaller than the TO-247 of the IGBTs. Better performances are obtained with smaller devices, increasing the power density of the converter. Considering also that the size of the output filter can be reduced by increasing the switching frequency thanks to the low switching losses of $\mathrm{SiC}$ devices, the advantages of employing the proposed modulation with a full SiC MOSFET ANPC converter are evident.

\section{REFERENCES}

[1] S. Araujo, P. Zacharias, and R. Mallwitz, "Highly efficient singlephase transformerless inverters for grid-connected photovoltaic systems," IEEE Transactions on Industrial Electronics, vol. 57, no. 9, pp. 3118-3128, Sep. 2010.

[2] D. Barater, G. Buticchi, A. Crinto, G. Franceschini, and E. Lorenzani, "Unipolar pwm strategy for transformerless pv grid-connected converters," IEEE Transactions on Energy Conversion, vol. 27, no. 4, pp. 835843, Dec 2012.

[3] T. Kerekes, R. Teodorescu, P. Rodriguez, G. Vazquez, and E. Aldabas, "A new high-efficiency single-phase transformerless pv inverter topology," IEEE Transactions on Industrial Electronics, vol. 58, no. 1, pp. 184-191, Jan 2011.

[4] B. Gu, J. Dominic, J.-S. Lai, C.-L. Chen, T. LaBella, and B. Chen, "High reliability and efficiency single-phase transformerless inverter for grid-connected photovoltaic systems," IEEE Transactions on Power Electronics, vol. 28, no. 5, pp. 2235-2245, May 2013.

[5] B. Ji, J. Wang, and J. Zhao, "High-efficiency single-phase transformerless pv h6 inverter with hybrid modulation method," IEEE Transactions on Industrial Electronics, vol. 60, no. 5, pp. 2104-2115, May 2013.

[6] H. Xiao and S. Xie, "Leakage current analytical model and application in single-phase transformerless photovoltaic grid-connected inverter," IEEE Transactions on Electromagnetic Compatibility, vol. 52, no. 4, pp. 902-913, Nov 2010.

[7] G. Buticchi, D. Barater, E. Lorenzani, and G. Franceschini, "Digital control of actual grid-connected converters for ground leakage current reduction in pv transformerless systems," IEEE Transactions on Industrial Informatics, vol. 8, no. 3, pp. 563-572, Aug 2012.

[8] A. Nabae, I. Takahashi, and H. Akagi, "A new neutral-point-clamped pwm inverter," Industry Applications, IEEE Transactions on, vol. IA-17, no. 5, pp. 518-523, Sep. 1981.

[9] T. Bruckner and S. Bemet, "Loss balancing in three-level voltage source inverters applying active npc switches," in Power Electronics Specialists Conference, PESC 2001, vol. 2, 2001, pp. 1135-1140 vol.2.

[10] J. Li, J. Liu, D. Boroyevich, P. Mattavelli, and Y. Xue, "Threelevel active neutral-point-clamped zero-current-transition converter for sustainable energy systems," IEEE Transactions on Power Electronics, vol. 26, no. 12, pp. 3680-3693, Dec 2011.

[11] D. Floricau, E. Floricau, and M. Dumitrescu, "Natural doubling of the apparent switching frequency using three-level anpc converter," in ISNCC 2008. International School on Nonsinusoidal Currents and Compensation, 2008., Jun. 2008, pp. 1-6.

[12] T. Bruckner, S. Bernet, and H. Guldner, "The active npc converter and its loss-balancing control," IEEE Transactions on Industrial Electronics, vol. 52, no. 3, pp. 855-868, Jun. 2005.

[13] D. Floricau, G. Gateau, M. Dumitrescu, and R. Teodorescu, "A new stacked npc converter: 31-topology and control," in Power Electronics and Applications, 2007 European Conference on, Sep. 2007, pp. 1-10.

[14] D. Floricau, G. Gateau, and A. Leredde, "New active stacked npc multilevel converter: Operation and features," IEEE Transactions on Industrial Electronics, vol. 57, no. 7, pp. 2272-2278, Jul. 2010.

[15] Y. Wang and R. Li, "Novel high-efficiency three-level stacked-neutralpoint-clamped grid-tied inverter," IEEE Transactions on Industrial Electronics, vol. 60, no. 9, pp. 3766-3774, Sep. 2013.

[16] D. Barater, C. Concari, G. Buticchi, E. Gurpinar, D. De, and A. Castellazzi, "Performance evaluation of a 3-level anpc photovoltaic gridconnected inverter with $650 \mathrm{v}$ sic devices and optimized pwm," in Energy Conversion Congress and Exposition (ECCE), 2014 IEEE, Sep. 2014, pp. 2233-2240. 
[17] J. Rodriguez, S. Bernet, B. Wu, J. Pontt, and S. Kouro, "Multilevel voltage-source-converter topologies for industrial medium-voltage drives," Industrial Electronics, IEEE Transactions on, vol. 54, no. 6, pp. 2930-2945, Dec 2007.

[18] P. Anthony and N. McNeill, "The efficient deployment of silicon superjunction mosfets as synchronous rectifiers," in International Conference on Power Electronics, Machines and Drives, PEMD 2014, 2014.

[19] M. Conrad and R. DeDoncker, "Avoiding reverse recovery effects in super junction mosfet based half-bridges," in Power Electronics for Distributed Generation Systems (PEDG), 2015 IEEE 6th International Symposium on, 2015.

[20] G. Busatto, G. Persiano, and F. Iannuzzo, "Experimental and numerical investigation on mosfet's failure during reverse recovery of its internal diode," IEEE Transactions on Electron Devices, vol. 46, no. 6, pp. 1268-1273, Jun 1999.

[21] A. Anthon, Z. Zhang, M. A. E. Andersen, and T. Franke, "Efficiency investigations of a $3 \mathrm{kw}$ t-type inverter for switching frequencies up to 100 khz," in International Power Electronics Conference, 2014. IPEC 2014, 2014, pp. 78-83.

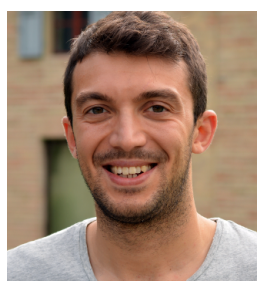

Davide Barater (S'11-M'14) was born in Italy on August 13, 1983. He received the Master's degree in Electronic Engineering in 2009 and the Ph.D. in Information Technologies in 2014 from the University of Parma, Italy. He was an honorary scholar at the University of Nottingham, U.K, during 2012. He is now working as research fellow at the Department of Information Engineering, University of Parma, Italy. His research area is focused on power electronics for renewable energy systems and motor drives. $\mathrm{He}$ papers, and holds one international patent. is author or coauthor of more than 20 international

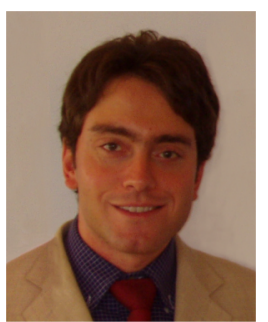

Carlo Concari (S'98-M'06) was born near Parma, Italy, in 1976. He received the M.S. degree in electronics engineering and the Ph.D. degree in information technologies from the University of Parma, Italy, in 2002 and 2006, respectively. Since 2014 he is an Associate Professor of power converters, electrical machines and drives with the Department of Information Engineering, University of Parma. His research activity includes power electronics, digital drive control, static power converters, and diagnostics of electric machines and drives. He is the author or coauthor of more than 50 international papers.

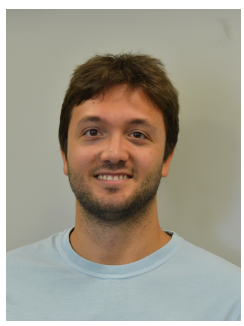

Giampaolo Buticchi was born in Parma, Italy, in 1985. He received the Master's degree in Electronics Engineering in 2009 and the Ph.D degree in Information Technologies in 2013 from the University of Parma, Italy. He is now working as a postdoctoral research associate at the Chair of Power Electronics, University of Kiel, Germany. His research area is focused on power electronics for renewable energy systems and grid integration.

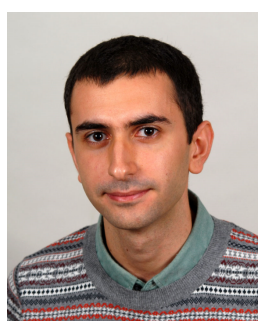

Emre Gurpinar (S'11) received the Bachelor of Science degree in electrical engineering from Istanbul Technical University, Istanbul, Turkey, in 2009 and the Master of Science degree in power electronics, machines and drives from the University of Manchester, Manchester, U.K. in 2010. After working as R\&D Power Electronics Engineer with General Electric, U.K., he joined the University of Nottingham, U.K. as a Ph.D. candidate in 2013. He is currently working on his Ph.D. thesis based on wide-bandgap semiconductor based renewable power converters. He was a visiting Ph.D. student at Department of Energy Technology, Aalborg University, Denmark between August 2015 and October 2015. His research interests include wide-band-gap power devices, high-frequency converters and renewable power systems.

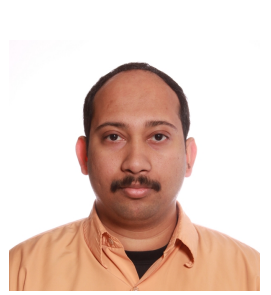

Dipankar De (M'11) completed his PhD from the Indian Institute of Science, Bangalore, India in 2011 with power electronics specialization. From 2011 to 2014, he was with the University of Nottingham, UK where he was involved in development of efficient energy storage systems and compact power converters design with wide band gap semiconductors.

Presently he is working as a research fellow with the Ryerson University, Canada for a sponsored project funded by Rockwell Automation, Canada. His research interests include power converter systems for renewable, energy storage and portable applications.

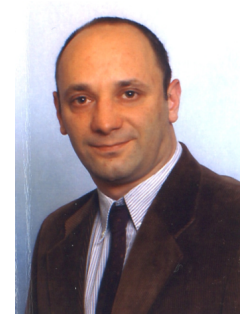

Alberto Castellazzi received the Laurea degree in physics from the University of Milan, Milan, Italy, in 1998 and the Ph.D. degree in electrical engineering from The Munich University of Technology, Munich, Germany, in 2004. He is an Associate Professor of power electronics with The University of Nottingham, Nottingham, U.K. He has been active in power electronics research and development for over 15 years and has had extensive collaborations with major European and international industrial research laboratories and groups on publicly and privately funded research projects. He has authored or co-authored over 130 papers published in peer reviewed specialist journals and conference proceedings, for which he also regularly acts as a reviewer. His research interests include characterization, modelling, application, packaging and cooling of power devices. He is a member of the Technical Programme Committee of the ISPSD, ESTC and ECCE-Asia. 